\title{
Long-term follow-up of two cases of pulmonary varicosity
}

\author{
TAKAYUKI SHIRAKUSA
}

From the Second Department of Surgery, School of Medicine, Fukuoka University, Fukuoka 812, Japan

\begin{abstract}
Shirakusa, T (1978). Thorax, 33, 653-656. Long-term follow-up of two cases of pulmonary varicosity. Before diagnosis by pulmonary angiography two cases of pulmonary varicosity, one in a young girl and the other in a middle-aged man, had been misdiagnosed as pulmonary tuberculosis. Both patients were asymptomatic and received no specific treatment. Six and four years later there was no radiological change in either patient. Previously reported cases are briefly reviewed.
\end{abstract}

Varicosity of the pulmonary vein is a rare condition usually discovered by chance. Since the first description (Puchet, 1843) 39 cases have been reported. The varix usually presents as a round shadow with a homogeneous density on the chest radiograph. The differential diagnosis is that of any coin shadow.

This paper reports two cases of pulmonary varicosity diagnosed angiographically and followed for six and four years respectively.

\section{Case reports}

CASE 1

A 16-year-old girl was diagnosed as having pulmonary tuberculoma in May 1971 because of a round shadow on the chest radiograph. From June 1971 to February 1972 she had been treated by antituberculosis chemotherapy, but the abnormal shadow showed no radiological change. In March 1972 she was admitted to Kyushu University Hospital for further examination. She was asymptomatic. Physically, the patient appeared to be healthy. The palpebral conjunctiva was slightly anaemic. Cyanosis, clubbing, and teleangiectasia were not seen. The PPD reaction was positive, and culture of sputum for acid-fast bacilli was negative. The electrocardiogram was normal. The chest radiograph showed a shadow $(3 \times 3.5 \mathrm{~cm})$ in the left hilar area (fig 1). The lateral tomogram showed a well-circumscribed lesion with a lobulated contour. A pulmonary angiogram via the femoral vein showed normal findings in the arterial phase and pooling of the contrast medium in the

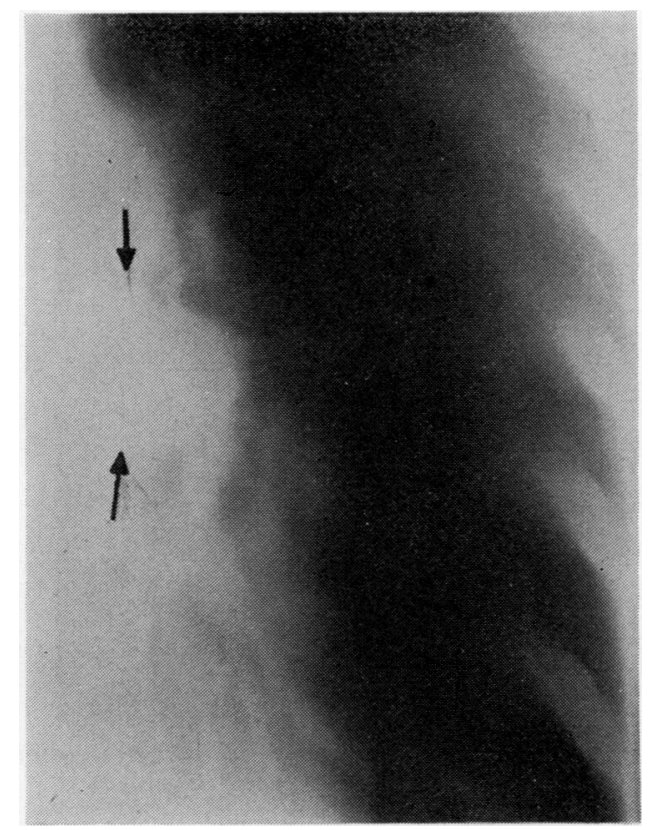

Fig 1 Case 1. Posteroanterior chest tomogram showing mass (arrow) at left hilum.

venous phase (fig 2). The lateral angiogram showed an abnormal dilatation (varix) of the left inferior pulmonary vein (fig 3 ). The patient was discharged without treatment. The chest radiograph in February 1978 showed no enlargement of the varix. She has never experienced haemoptysis or chest pain. 


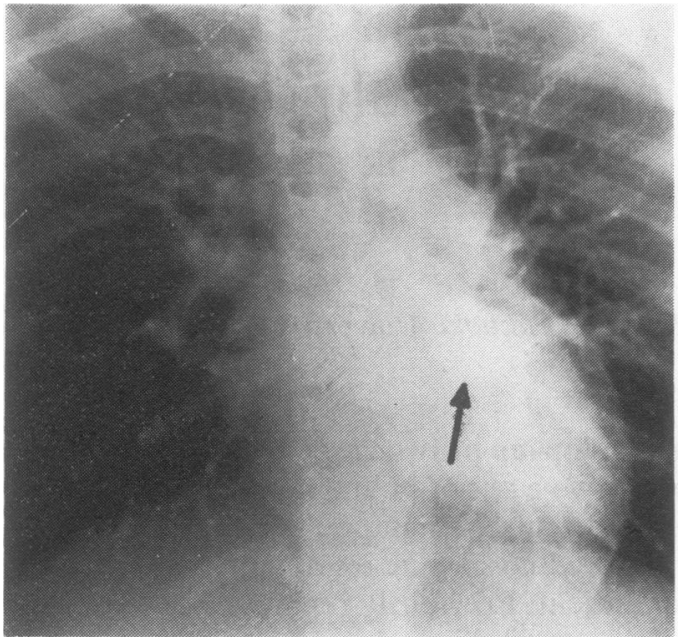

Fig 2 Case 1. Posteroanterior angiogram showing varix (arrow) of pulmonary vein.

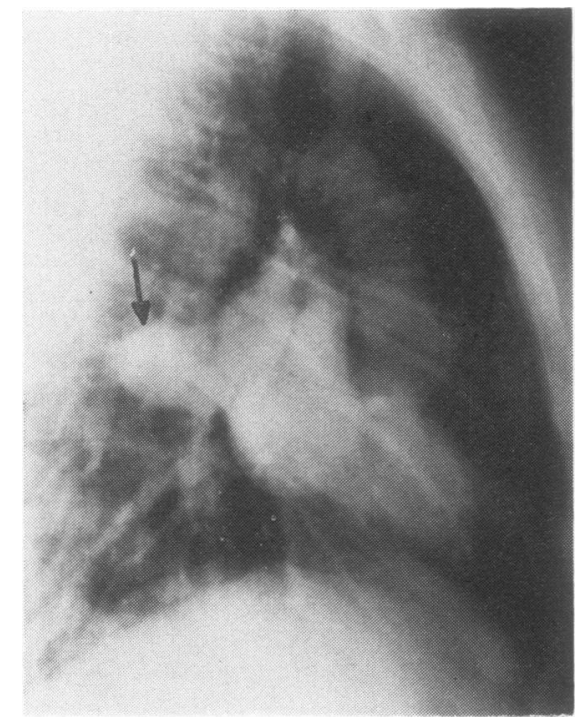

Fig 3 Case 1. Lateral angiogram showing retention of contrast medium at varix (arrow) of inferior pulmonary vein.

CASE 2

A 53-year-old man, who had had annual chest radiographic examinations since he was 38 because of an abnormal chest shadow, was admitted to Kyushu National Cancer Centre Hospital in December 1973. Before admission he had been treated by antituberculosis chemotherapy in another hospital, but there was no radiological change in the size of the lesion. He had no symptoms and no cyanosis and clubbing. Cultures of sputum for acid-fast bacilli were negative. The electrocardiogram was normal. The routine chest film and posteroanterior tomogram showed an irregularly contoured shadow at the right hilum (fig 4). The pulmonary angiogram showed a normal arterial phase and a saccular dilatation of a branch of a pulmonary vein (fig 5). It indicated that there was a varix at the central end of the right middle lobe vein. The patient was discharged without treatment, and he remains well. There has been no radiological change in the shadow.

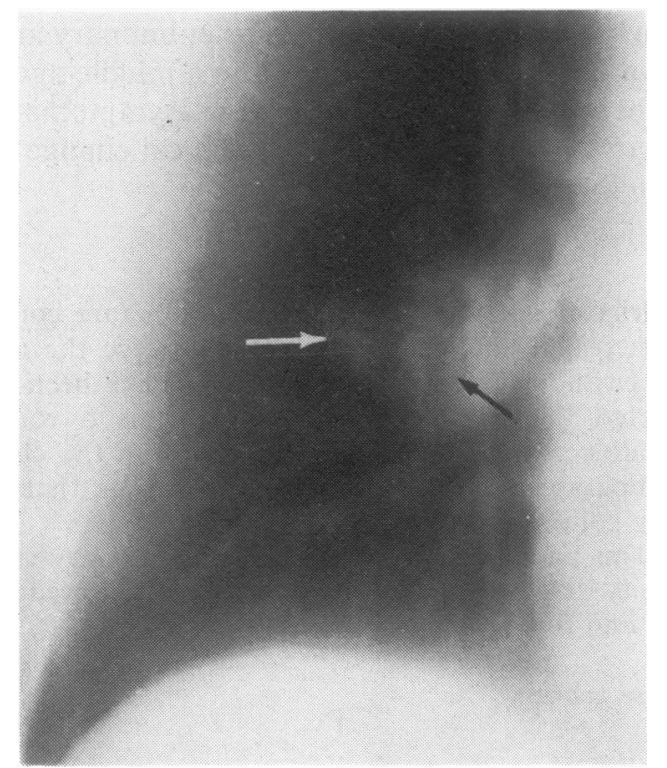

Fig 4 Case 2. Posteroanterior chest tomogram showing an irregular mass (arrow) at right hilum.

\section{Discussion}

Pulmonary varicosity was first discovered at necropsy (Neiman, 1934). In 1951 Mouquin et al $\tilde{N}$ diagnosed this disease by angiography in a living patient. Since then, more than 30 cases have been 0 reported. This disorder occurs in any decade $\omega$ (from the new-born to 69 years), but most cases are between the ages of 30 and 45 . The sex inci- 0 dence is equal. Symptoms and clinical signs are $\mathbb{D}$ unusual, but a few cases of haemoptysis (Papa- ? michael et al, 1972) or dyspnoea (Klinck and Hunt, 0 1933; Gottesman and Weinstein, 1959) have been reported. A fatal case due to rupture of the varix $\cong$

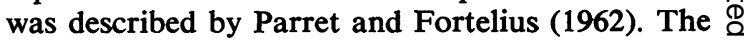
varicosity may be congenital in origin, and often $\bar{O}$ there are other abnormalities in the lungs (Kozuka 


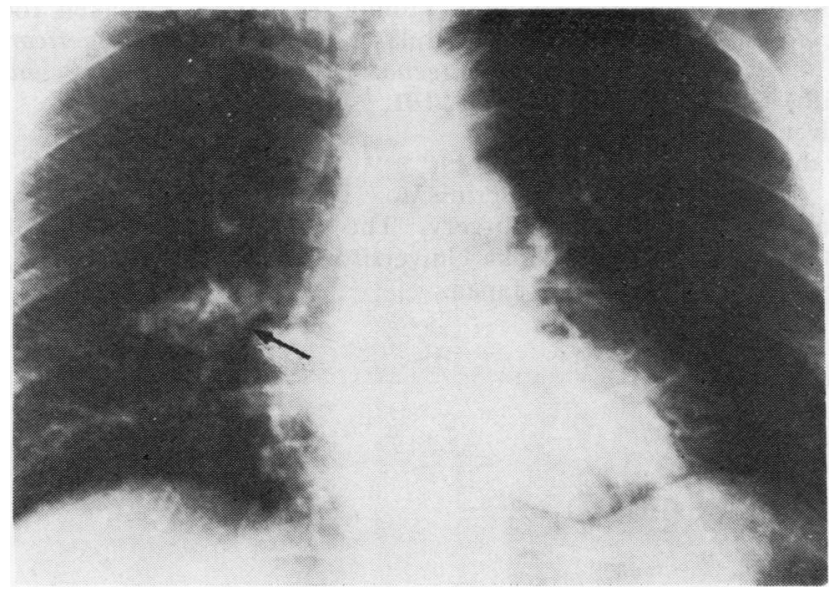

Fig 5 Case 2. Pulmonary angiogram of venous phase showing varix (arrow).

and Nozaki, 1968), the heart (Bryk and Levin, 1965), or large blood vessels (Rizk et al, 1970). In the present cases symptoms, signs such as cyanosis or clubbing, or other anomalies were not found.

Varix has to be differentiated from pulmonary arteriovenous fistula, pulmonary tuberculosis, carcinoma, and mediastinal tumour (Steinberg, 1967). On the chest radiograph the lesion often shows a lobulated but well-circumscribed contour. It is often discovered in the right lung, especially in the upper lobe, although in one of the present cases the lesion was located in the middle lobe and in the other in the superior segment of the left lower lobe. The lesions can usually be seen mingling with hilar vessels on posteroanterior tomograms. It can be easily differentiated from lung carcinoma by bronchographic or bronchoscopic examination. The author's cases had been treated as pulmonary tuberculosis in other hospitals before admission, and in case 2 the patient had been followed for 14 years. Differentiation from tuberculosis might have been achieved by the careful observation of posteroanterior or lateral tomograms. For the differentiation from arteriovenous fistula, angiography is necessary, though the presence of clubbing, cyanosis, and a vascular murmur would suggest the presence of arteriovenous fistula rather than varix (Arnett and Patton, 1976). In varicosity, the opacification by angiography is apparent in the venous phase only, whereas in arteriovenous fistula it also occurs in the arterial phase. The opacification may develop and fade slowly in the varicosity. Arnett and Patton (1976), in their review, found that four patients had been subjected to unnecessary diagnostic thoracotomy because angiography had not been performed.
Treatment is usually unnecessary but annual chest radiographs are advisable. The two cases reported here showed no change on recent radiographic re-examination. However, in the case of a reported haemoptysis or systemic embolism, resection of the lobe or segment might be indicated (Papamichael et al, 1972).

I thank Professor Inutsuka, School of Medicine, Fukuoka University, for his advice.

\section{References}

Arnett, J R, and Patton, R M (1976). Pulmonary varix. Thorax, 31, 107-112.

Bryk, D, and Levin, E J (1965). Pulmonary varicosity. Radiology, 85, 834-837.

Gottesman, L, and Weinstein, A (1959). Varicosity of the pulmonary veins. A case report and survey of the literature. Diseases of the Chest, 35, 322-327.

Klinck, G H, and Hunt, H D (1933). Pulmonary varix with spontaneous rupture and death. Archives of Pathology, 15, 227-237.

Kozuka, T, and Nozaki, T (1968). A pulmonary vein anomaly: unusual connection and tortuosity of the right lower lobe vein. British Journal of Radiology, 41, 232-234.

Mouquin, M, Hébrard, H, Damasio, R, Jouvet, P, Durand, $M$, and Piéquet, J (1951). Varice du poumon diagnostiquée par l'angiocardiographie. Bulletins et Mémoires de la Société Médicale des Hôpitaux de Paris, 67, 1091-1094.

Neiman, B H (1934). Varix of pulmonary vein. The American Journal of Roentgenology, 32, 608-612.

Papamichael, E, Ikkos, D, Alkalais, $K$, and Yannacopoulos, J (1972). Pulmonary varicosity associated with other congenital abnormalities. Chest, 62, 107109. 
Parret, L, and Fortelius, P (1962). Ruptured aneurysm of the pulmonary vein. Acta Tuberculosa Scandinavica, 41, 53-55.

Puchet (1843): quoted by Gimes, B, and Horváth, F (1958). Uber die Varikosität der Pulmonalvene. Fortschritte auf dem Gebiete der Roentgenstrahlen und der Nuklearmedizin, 89, 545-548.

Rizk, G, Melhem, R, Dagher, I (1970). Bilateral pulmonary varicosities associated with coarctation of the aorta. Thorax, 25, 97-100.
Steinberg, I (1967). Pulmonary varices mistaken for pulmonary and hilar disease. The American Journal of Roentgenology, Radium Therapy and Nuclear Medicine, 101, 947-952.

Requests for reprints to: Dr Shirakusa, Laboratory of Thoracic Surgery, The Second Department of Surgery, Fukuoka University Hospital, 34, Nanakuma, 814 Fukuoka Japan. 